\title{
Consumo en mercados alternativos para baja renta Un estudio de la Feria de Parangaba - Brasil
}

\author{
Verónica Peñaloza \\ Universidade Estadual do Ceará, Fortaleza, Brasil. \\ Email: veronica.penaloza@uece.br \\ Marianela Denegri Coria \\ Universidad de la Frontera, Temuco, Chile. \\ Email: marianela.denegri@ufrontera.cl

\section{Lucas Lopes Ferreira de Souza} \\ Universidade Estadual do Ceará, Fortaleza, Brasil. \\ Email: lucaslfssouza@gmail.com

\section{Felipe Gerhard Paula Sousa} \\ Universidade Estadual do Ceará, Fortaleza, Brasil. \\ Email: felipegerhard.rns@gmail.com
}

\begin{abstract}
Resumen: Mediante la complementación entre una mirada cuantitativa y el rescate de los significados cualitativos, este trabajo pretende contribuir al conocimiento del consumidor de mercados alternativos. Un hallazgo importante es que la diversión crea un valor agregado importante en el momento de decidir el lugar donde se harán las compras. El comercio a pequeña escala, la atención personalizada y el cultivo del vínculo con el cliente son una enseñanza que se deriva de la observación de la realidad de las ferias y que puede ser un referente importante para la planeación de negocios con estos sectores emergentes.
\end{abstract}

Palabras clave: Consumo hedonista, consumo utilitarista, consumo de Baja Renta, mercados alternativos.

\section{Consumption in alternative markets for low incomes A study at Parangaba's Fair - Brasil}

\begin{abstract}
Considering quantitative and qualitative methods as approaches that have the potential to complement each other, this study aims at understanding the consumers of alternative market. One of the findings of the research is that the option for fun generates an important agregated value that affects place-of-purchase decision. Small-scale commerce, personalized attention and bond with customers are features originated from the reality observed in fairs, which may be a relevant reference for business planning in those emerging sectors.

Keywords: Hedonic consumption, utilitarian consumption, Low-Income consumption, alternative markets.
\end{abstract}




\section{Consumo em mercados alternativos de baixa renda Um estudo da Feira Parangaba - Brasil}

Resumo: Por complementação entre um olhar quantitativo e o resgate dos significados qualitativos, este trabalho visa contribuir para a o conhecimento do consumidor de mercados alternativos. Um achado importante é que a diversão cria um valor acrescentado significativo para decidir onde as compras são feitas. $\mathrm{O}$ comercio em pequena escala, atenção pessoal e crescente relacionamento com o cliente é uma lição a ser aprendida a partir da observação da realidade das feiras e pode constituir um referente importante para o planejamento de negócios com estes sectores emergentes.

Palavras-chave: Consumo Hedonista; Consumo utilitarista; Consumo de Baixa Renda; Mercados alternativos.

***

\section{Introducción}

Los mercados alternativos han sido tradicionalmente un objeto de estudio de áreas como la economía y la sociología, tanto desde el punto de vista del impacto sobre la actividad económica (evasión fiscal por ejemplo) como desde el punto de vista de la naturaleza social de sus participantes (comportamiento de relaciones entre locatarios, por ejemplo). Sin embargo, ha sido de relativamente poco interés para el área de marketing, área que se ha centrado tradicionalmente en el estudio de los mercados formales. Desde esta perspectiva, se ha estudiado el lado de la oferta de los mercados, pero con escaso interés en el lado de la demanda, menos aún en el comportamiento del consumidor.

Según Sherry (1990) los mercados no tradicionales existen en todo el mundo, sin embargo, en las regiones menos desarrolladas estos centros de comercio, muchas veces informales, forman parte de la vida cotidiana de los sectores más pobres y a menudo son una forma de acceso de esta capa de la población a productos más baratos que estén al alcance de su presupuesto.

Hasta finales de los años 90, eran muy pocas las investigaciones sobre el consumo en las clases sociales más bajas, ello coincidía con el bajo interés de las empresas en invertir en productos y servicios dirigidos a este segmento. No obstante, después del estudio de Prahalad y Hart (2002) quienes presentan a este segmento como poseedor de un gran potencial de consumo, las empresas nacionales y multinacionales comenzaron a mostrar interés en direccionar productos y servicios específicos para estos grupos de consumidores. Sin embargo, a pesar de la importancia que el tema ha ido cobrando en los medios académicos y de comunicación, poco se conoce sobre los mercados destinados a estos segmentos lo que sumado al bajo interés del poder político local, esto se traduce en una ausencia casi total de información oficial y sistematizada sobre ellos y sus particularidades. 
Desde la Edad Media, los únicos locales de comercio a los que tenía acceso la población eran los pequeños mercados locales estrictamente organizados para satisfacer las necesidades de consumo de la burguesía y las ferias itinerantes que podían tener un carácter local, regional e incluso internacional. Estos eran lugares donde periódicamente convergían comerciantes de diversos objetos y para los cuales cada ciudad organizaba espacios permanentes, ya sea pequeñas tiendas techadas o casetas donde se ubicaban los distintos comerciantes. Su estructura era similar a los mercados locales pero difería en que no atendían todos los días en el mismo lugar, aspecto que aún hoy conservan algunas de las ferias libres itinerantes que atienden a la población distintos días de la semana en distintos lugares de la ciudad (Cuellar \& Parra, 2001; Calderón, 2012). Hoy en día, muchas de estas estructuras antiguas que subsistieron durante varios siglos han sido progresivamente sustituidas por modernos hipermercados y centros comerciales, sin embargo muchas de ellas aún subsisten en localidades rurales, ciudades menores o bien en algunos barrios más apartados del centro. Para Pandolfo (1987), esta práctica comercial común de las sociedades del Tercer Mundo, continúa desafiando a la moderna economía. Según el autor, funcionando en espacios abiertos (generalmente plazas o calles transversales) y obedeciendo a una periodicidad regular, las ferias, representantes de los mercados alternativos en ese estudio, se constituyen en locales de circulación, distribución y consumo de bienes y servicios donde son comercializados además de géneros alimenticios de primera necesidad, muchos otros tipos de productos tal como funcionaban los mercados locales inicialmente y las propias ferias itinerantes.

En las regiones menos desarrolladas, las ferias ofrecen a los consumidores de bajos ingresos varias alternativas de consumo que no se encuentran en los mercados tradicionales, así puede encontrarse desde la venta de productos usados hasta animales. Se constituyen así en un mundo diverso incluso extravagante y colorido donde los consumidores encuentran un espacio para expresar sus necesidades de consumo. Por ello, aunque la naturaleza de estos mercados es eminentemente mercantil, también funcionan como centros culturales y de ocio donde el consumo muestra tanto su lado hedónico como utilitario. Este es el caso de la Feria de Parangaba, o Feria de los Pájaros como también es conocida, un gran mercado de comercio popular e informal, tal vez uno de los mayores centros de comercio al aire libre y la mayor feria popular de la región de Ceará, nordeste brasileño.

En vista de la carencia de aportes teóricos concernientes a los mercados alternativos y a los consumidores de baja renta, el propósito de este trabajo es contribuir al conocimiento de los mercados alternativos en relación al comportamiento de compra y perfil de los consumidores que asisten a ellos, tomando como estudio de caso a la Feria de Parangaba. Mediante una aproximación mixta cualitativa y cuantitativa, se pretende, por una parte conocer la representación social de los consumidores acerca de la Feria de los Pájaros, y por otra, a través de la investigación cuantitativa describir el perfil de los consumidores en términos de ingresos, para determinar si 
efectivamente constituyen un segmento de baja renta. Se espera además, identificar las razones para frecuentar la Feria, los productos realmente demandados y las características del valor de compra en términos de consumo hedonista y utilitarista.

Este trabajo se estructura en cuatro sesiones, además de esa introducción. Inicialmente será abordado el contexto empírico en cuestión, así como los principales aspectos teóricos de los mercados alternativos; en seguida será presentada una síntesis sucinta de estudios relacionados al consumo hedónico e utilitario. En consecuencia serán tratados los aspectos metodológicos de la investigación. Por final, serán inferidos los resultados y presentadas las conclusiones, así como las limitaciones y sugerencias para investigaciones futuras.

\section{Referencial Teórico}

En el referencial teórico se pretendió trazar un marco conceptual que diera sustentación a la investigación a partir de algunos tópicos específicos: Mercados alternativos, representados en la Feria de Parangaba y la existencia de valores de consumo hedónico y utilitario poco estudiados en mercados de este tipo.

\section{Mercados alternativos: Feria de Parangaba o Feria de los Pájaros}

Situada en Parangaba, al borde de la laguna del mismo nombre, este barrio, está ubicado en la región Este de la ciudad, siendo uno de los principales aglomerados de comercio no tradicional de Fortaleza, capital de Ceará. Con más de 20 años de existencia activa, la Feria de Parangaba, se convirtió en "el mayor ejemplo de coqueteo entre la informalidad y la ilegalidad” en Fortaleza (Editorial Verdes Mares, 2010). La feria callejera que tuvo su inicio en la época colonial en la que se vendía ganado, en la actualidad vende todo tipo de productos, desde alimentos a vestuario, desde fantasías hasta repuestos de automóviles, pasando por el comercio ilegal de animales, pájaros y aves, siendo esto último lo que dio lugar a su nombre.

Para Olavarrieta, Manzur, Hidalgo y Farías (2008), los mercados alternativos se caracterizan por tener bajas barreras de entrada, baja regulación y porque no se requiere de un gran capital para instalar el negocio. Estos funcionan a pequeña escala, haciendo uso intensivo de mano de obra y algunos son de propiedad familiar. En este caso, a pesar de existir un registro formal de los comerciantes de la feria (más de 2000 registrados) y un proyecto de urbanización del sector en el municipio de la ciudad, aun así no es posible estimar la dimensión de los negocios informales existentes en ella. Así, la informalidad, junto con los aspectos ilegales, constituye la estructura predominante en la Feria de los Pájaros. En el sector informal, el comportamiento del consumidor difiere del realizado en el sector formal. En las ferias y otros mercados informales, el comportamiento de los consumidores está menos estandarizado, es más libre y por lo tanto permite una 
oportunidad de experimentar, que puede ser liberadora para algunos consumidores, y amenazadora para otros, coexistiendo lo "peligroso" o "emocionante". Los compradores más frecuentes de estos mercados sienten que es divertido encontrar ofertas y perciben la tención como más amable y cercana a la vez que disfrutan de la variedad de productos disponibles (Sherry, 1990, Belk, Wallendorf \& Sherry, 1988). De hecho, según Molina (2010), la confianza es un elemento de comportamiento importante que orienta la conducta entre consumidores y feriantes, que entrelaza y fundamenta la gran red de interacciones que es la feria. En cambio, otros compradores menos asiduos de estos mercados perciben los productos como de baja calidad, falta de seguridad y están preocupados si los productos son robados o si existe evasión fiscal (Olavarrieta et al., 2008). Es el caso de la Feria de los Pájaros, donde los precios son bajos, no están expuestos y siempre hay disponibilidad de negociar y regatear. Caminar por los callejones de la Feria debe ser, ante todo, un ejercicio de atención y mucho cuidado dado que la venta de bienes robados, falsificados y de comercialización prohibido es evidente (Editora Verdes Mares, 2010).

Aunque una de las principales características de los mercados alternativos son los precios bajos, estos no son el único punto atractivo de estos mercados. Muchos compradores perciben estos mercados como una experiencia agradable de compra, como un lugar para divertirse y socializar, (Sherry, 1990; Belk, Wallendorf \& Sherry, 1988). Por otra parte, aunque la búsqueda de gangas (productos de bajo precio) puede ser más intensa en los segmentos socioeconómicos de bajos ingresos, la evidencia empírica sobre el perfil socioeconómico del comprador de los mercados no tradicionales es controversial. Algunos autores sugieren que los compradores de los mercados informales son en su mayoría los segmentos socioeconómicos más bajos, mientras que otros autores sugieren que los segmentos de alto nivel socioeconómico también frecuentan este tipo de mercado (Olavarrieta et al., 2008).

Para Sherry (1990), los mercados alternativos, incorporan la tensión inherente de lo formal-informal y lo económico-festivo en mayor grado que en los mercados tradicionales. La estructura informal es vista como menos oficial (a menudo ilegal), menos controlada, menos racionalizada, reactiva y de naturaleza transitoria. En ellos coexiste la función económica que se caracteriza por las nociones de racionalidad y utilidad con la función festiva y hedonista que es experiencial por naturaleza.

En resumen, a pesar de estar presentes en formas, espacios y culturas diferentes, los mercados no tradicionales, tienen ciertas estructuras similares de funcionamiento: una variedad de productos comercializados, actividades legales y no legales, consumidores de altos y bajos ingresos, más predominantemente estos últimos, así como también la presencia de comportamientos de compras tanto hedónicos como utilitarios (Belk, Wallendorf \& Sherry, 1988; Sherry, 1990; Zinkhan, Fontenelle \& Balazs, 1999; Companion, 2008; Lauermann, 2012). Así, como bien resume Sherry (1990), la estructura de estos mercados se puede desdoblar en dos dimen- 
siones dialécticas, siendo la dimensión estructural representada por la dialéctica formal-informal, y la dimensión funcional representada por la dialéctica económica y festiva.

\section{Consumo hedónico y utilitario}

Desde la perspectiva de la teoría económica tradicional, los consumidores hacen sus elecciones de forma racional y objetiva, buscando el menor dispendio monetario. Este tipo de consumo es racional y eficiente. El valor utilitario de una compra se basa en la utilidad que aquella compra tiene para el consumidor, en ella los consumidores no buscan una experiencia, sólo van con la intención de encontrar el producto que buscan, a un buen precio y que conlleve el mínimo esfuerzo. Assí, el valor utilitario es obtenido a través de la eficiencia en que se generó el proceso de compra: entrada, comprar el producto/servicio correcto, salida y esfuerzos o recursos utilizados (Babin, Darden \& Griffin, 1994).

Las características emocionales que motivan el consumo sólo comenzaron a ser más estudiadas a partir de los años 80 a partir de las investigaciones de Holbrook y Hirschman (1982). Para estos autores, la noción de consumo hedónico, salía al encuentro del consumo utilitario, porque era motivada por aspectos multisensoriales, emotivos y de fantasías del consumidor. Este tipo de consumo se relaciona con el valor subjetivo percibido por el consumidor en la experiencia de compra y que por tanto pueden variar en función del contexto de cada uno. Así, la decisión de compra es sólo un pequeño componente de entre todos los involucrados en la experiencia de consumo en general (Holbrook \& Hirschman, 1982; Babin, Darden \& Griffin; 1994; Hausman, 2000).

Autores como Bardhi y Arnould (2005), Lopes, Teixeira, Hernandez y Moretti (2010), están de acuerdo en que en una experiencia de compra pueden coexistir el consumo utilitario y el consumo hedónico porque, aun cuando son valores diferentes, no son mutuamente excluyentes dado que abordan distintas características. El enfoque utilitario se refiere a los aspectos racionales, objetivos y económicos, mientras que el hedonista se centra en las características psicológicas, simbólicas y emocionales del consumo (Hernández, 2009).

\section{Método}

Según Rocha e Silva (2009), la investigación con los consumidores de baja renta es un desafío, ya que los métodos tradicionales se han desarrollado para los consumidores con mayores niveles de educación, en especial los de tipo encuestas y cuestionarios de investigación que requieren el uso de escalas. Pensando en estas dificultades, la presente investigación incorpora un abordaje tanto cualitativo como cuantitativo en forma de dos estudios complementarios. El primero de ellos, de carácter cualitativo, pretendía conocer cuál era la representación social que los consumidores tenían de la Feria y el segundo, de carácter cuantitativo, pretendía obtener 
informaciones más amplias sobre el perfil de este tipo de consumidor y el tipo de consumo predominante en la Feria (hedónico / utilitario).

\section{Estudio1: Test de Evocación de Palabras}

\section{Participantes}

Se trabajó con una muestra intencionada de carácter incidental compuesta por 130 sujetos, de ambos sexos 104 (80\%) hombres y 26 (20\%) mujeres, todos mayores de 15 años.

\section{Técnica de recolección de datos}

Para esta etapa del estudio se utilizó la técnica de evocación de palabras. Esta técnica ha sido utilizada en el área de ciencias sociales y en ella el investigador solicita a los entrevistados que mencionen un determinado número de palabras relacionadas a una expresión inductora. Permite la identificación de los elementos relacionados con el objeto de investigación, que se podría perder con otro tipo de análisis, y permite el examen combinado de una información de carácter colectivo: la frecuencia de las evocaciones, con un dato de carácter individualizado: el orden de la evocación. El test está basado en la teoría de las representaciones sociales introducidas por Serge Moscovici en 1961, pero no se agota en ella (Vergara, 2008). Las representaciones sociales pueden ser definidas como categorías de pensamiento que se refieren al contenido simbólico de los fenómenos basados en la realidad cotidiana. Determinadas por el lenguaje habitual, son una forma de conocimiento práctico de la construcción de una realidad común para un grupo social.

\section{Procedimiento y análisis}

Para la evocación de palabras se utilizó la expresión inductora "Feria de Parangaba". Se pidió a los encuestados que expresaran las primeras cuatro palabras sueltas que se les ocurrieran en relación a esta expresión y a continuación, se le preguntó cuál era el orden en términos de importancia que concedían a estas palabras.

Para el análisis, en primer lugar se clasificaron las palabras en categorías con base en un sentido próximo y fueron excluidas las palabras o frases mencionados sólo una vez y no categorizadas y luego se procedió a calcular la frecuencia de las categorías y el cálculo de orden promedio de evocación (OME), teniendo en cuenta la posición en la que la palabra o expresión fue jerarquizada por el entrevistado. Los resultados se representaron gráficamente en un diagrama de cuatro cuadrantes.

\section{Estudio 2: Survey}

Para el segundo estudio se utilizó una encuesta aplicada a una muestra no probabilística por conveniencia de 301 personas. Una muestra 
probabilística representativa de la Feria no sería posible, ya que no existe información documentada sobre los consumidores del lugar.

El instrumento se compone de 3 partes. La primera parte corresponde a la escala modificada Babin, Darden y Griffin (1994) en escala Likert de 7 puntos. La elección de esta escala se debe al hecho de esta mide el valor utilitario y el valor hedónico en una situación de consumo al por menor, y también porque ha sido validado en varias ocasiones (Jones, Reynolds \& Arnold, 2006). En esta investigación, utilizamos la escala adaptada por Lopes et al. (2010), aplicada en estudios con consumidores brasileños y en estudios con consumidores de bajos ingresos en la región de Fortaleza - CE (Peñaloza, Gordiano \& Quezado, 2011).

La segunda parte de la encuesta constaba de 11 preguntas abiertas acerca de los elementos que la persona demanda, las razones por la que compra en la Feria, otros lugares (y razones) en los que acostumbra comprar. La tercera parte del cuestionario correspondió a las cuestiones sociodemográficas, de género, edad, educación e ingreso familiar.

Los cálculos estadísticos se realizaron con la ayuda del software Statistical Package for the Social Sciences (SPSS) versión 18.0, utilizando los módulos de estadística descriptiva, análisis factorial y la prueba t.

\section{Resultados}

\section{Estudio 1}

Fueron evocados 180 palabras o expresiones, reunidas posteriormente en 13 categorías semánticas. Se excluyeron 32 palabras o frases mencionadas sólo una vez, no siendo categorizadas, expresiones tales como "Dios en el cielo", “circo de horrores” o palabras como "fogueteiro”, "salud” o "paz”. Considerando las recomendaciones de la literatura (Sherry, 1990) se utilizaron inicialmente como referencia 6 categorías. Estas fueron: "Entretenimiento/Ocio", "Variedad/Diversidad”, “Utilitarismo”, “Mercado”, “Informalidad/Desorganización” y “Ilegalidad”. Las 3 primeras categorías se asociaron con la dimensión funcional, “Económico-Festiva” señalada por Sherry (1990), así como a los conceptos de consumo hedónico/utilitario. Las otras categorías se asociaron con la dimensión estructural "Formal-Informal”. Además de estas categorías, previamente delimitadas que emanaron de la literatura, fueron creadas otras siete categorías a partir de la lista de términos que se presentaron en el desarrollo de la prueba. Estas fueron: “Empleo/Ingreso”, “Inseguridad/Miedo”, “Productos Comercializados, “Aglomeración/Amontonamiento”, “Atributos Positivos”, “Atributos Negativos” y “Alimentación”.

La figura 1 esquematiza los resultados de la categorización. En el plano cartesiano, los puntos ubicados arriba indican una mayor frecuencia y los puntos ubicados más a la izquierda indican mayor prioridad en la evocación. Los puntos (x, y) equivalen a (OME, frecuencia). El cuadrante 
superior izquierdo (II) se refiere a la representación central de la Feria de Parangaba. En este cuadrante están las evocaciones de mayor frecuencia y mayor importancia o prioridad. Como puede observarse, la categoría 'Entretenimiento/Ocio” es una representación más consensual y fundamental de la Feria. El cuadrante inferior izquierdo (III) está formado por las categorías mencionadas con menos frecuencia, pero evocadas más prontamente, son las categorías nombradas por menos personas, pero más importantes en términos de prioridad. En este cuadrante se encuentran las categorías "Variedad/Diversidad”, “Utilitario”, además de la categoría de “Empleo/Ingresos”. Por lo tanto, el lado izquierdo de la Figura 1, de mayor prioridad en la evocación puede ser asociado a la dimensión funcional de Sherry (1990). En el cuadrante superior derecho (I), tenemos las categorías más frecuentes, pero con menor importancia de evocación, "Mercado", "Productos Comercializados", "Informalidad/ Desorganización”, “Atributos Positivos” y "Inseguridad/Miedo.” El cuarto cuadrante inferior derecho (IV) reúne los términos y evocados menos frecuentes y en lugares menos prioritarios. Estos son considerados los elementos más periféricos de la representación. Aquí tenemos las categorías "Aglomeración/ Amontonamiento", “Comida” y “Atributos Negativos” de la Feria. En cuanto a la categoría de "Ilegalidad” definida en función de la revisión de la literatura, el concepto no aparece tan claramente como significado contrario a lo legal, las palabras evocadas estaban más en el sentido de mentira, engaño, o trucos ilegales para tomar ventaja de la situación. Por lo tanto, se decidió cambiar el nombre de la categoría a la de "Pilleria". El lado derecho de la misma, puede estar asociada con la dimensión estructural "formal-informal” de los mercados alternativos de acuerdo con el diseño propuesto por Sherry (1990).

\section{Figura 1. Distribución en cuadrantes de la frecuencia y orden promedio de evocación de las categorías sometidas a análisis.}

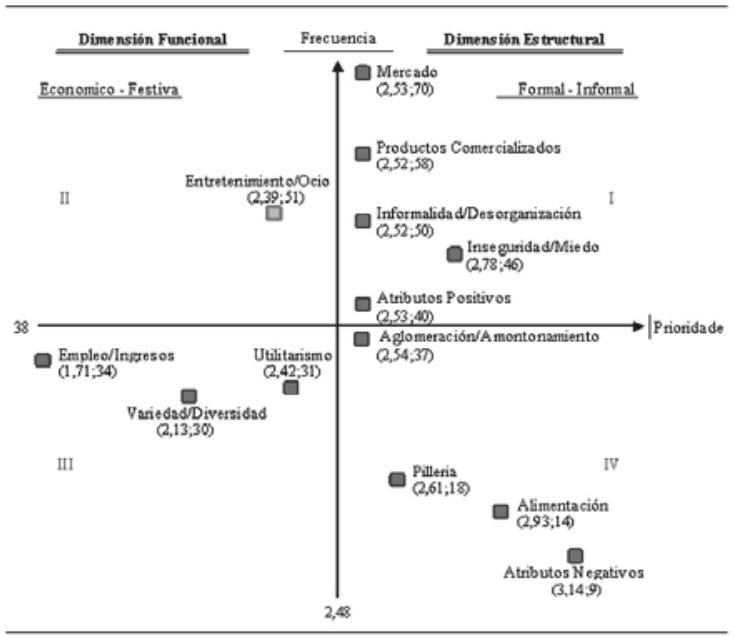

Fuente: elaboración propia. 


\section{Estudio 2}

\section{Resultados socio-demográficos}

Respondieron el cuestionario, 301 personas, 244 hombres (81,1\%) y 57 mujeres (18,9\%), con edades que fluctúan entre 15 y 68 años, con un promedio de 38 años. A pesar de que los datos parecen revelar una muestra descompensada en cuanto a género, es importante recordar que, no obstante la ausencia de estadísticas, en la Feria de los Pájaros la mayoría de los frecuentadores son hombres, a diferencia de las ferias convencionales, donde predomina el género femenino. En cuanto al perfil socio-económico, como consta en la Tabla 1, los datos de ingresos familiares y la educación, dan evidencia de que las personas que asisten a la Feria realmente pertenecen a los sectores más populares de la población. Con respecto a los ingresos familiares, medido en salarios mínimos (sm), 50\% de los encuestados tienen ingresos de hasta $3 \mathrm{sm}$, siendo el promedio de 3,68sm. El cuartil más pobre tiene un ingreso de hasta $1,5 \mathrm{sm}$ y el cuartil más rico tiene un ingreso superiora $4 \mathrm{sm}$. En cuanto a la escolaridad, el 21,3\% ha terminado la educación primaria, el 40,2\% no ha terminado la escuela secundaria, el 52,8\% dice haber completado la escuela secundaria, el 5,6\% cuenta con estudios superiores.

Tabla 1. Características de los consumidores

\begin{tabular}{lrr}
\hline \hline & \multicolumn{1}{c}{ Total } & $\%$ \\
\hline Género & 244 & 81,1 \\
\hline Maculino & 57 & 18,9 \\
Femenino & & \\
Renta & & \\
\hline $1^{\circ}$ Cuartil & hasta $1,5 \mathrm{sm}$ & 25 \\
$2^{\circ}$ Cuartil & entre 1,51 y $3 \mathrm{sm}$ & 25 \\
$3^{\circ}$ Cuartil & entre 3,1 y $4 \mathrm{sm}$ & 25 \\
$4^{\circ}$ Cuartil & acima de $4 \mathrm{sm}$ & 25 \\
\hline
\end{tabular}

Fuente: Elaboración propia.

En general se observa que los frecuentadores de la Feria son predominantemente hombres, con una edad cercana a 40 años, media educación, bajos ingresos que viven en su mayoría en los barrios próximos y se orientan a un valor de compra un poco más utilitario que hedónico. Suelen buscar la Feria principalmente por el bajo precio y por distracción, pero también por la variedad de productos, que van desde celulares y electrónicos, hasta productos alimenticios. 
Cuando se le preguntó acerca de las razones para asistir a la Feria, de las 512 respuestas libres, el precio fue la razón más citada (32,2\%), seguida de distracción/entretenimiento/ocio (20,7\%) y razones diversas (37,6\%). Otras razones citadas eran, gustos/preferencias (9,6\%), la calidad, el servicio, personalizado y de proximidad, entre otros. Consultados sobre los ítems que buscaban, algunas personas no sabían responder o se sintieron incomodadas con la pregunta, lo que llevó a un alto porcentaje $(42,5 \%)$ de no respuestas, así como otros respondieron, "de todo" sin especificar un elemento en particular lo que podría asociarse a la naturaleza ilegal de algunos productos que se venden allí, lo que llevo a los participantes a no reconocer que es ese tipo de productos los que buscan. Entre los artículos mencionados un mayor número de veces, fueron electrónicos, celular, géneros alimenticios, vestuario y muchos consumidores $(36,88 \%)$ no compran en otros lugares. Dentro de los que reconocieron comprar en otros lugares, identificaran principalmente: el centro comercial de la ciudad (23,01\%), tiendas (18,58\%), centros comerciales $(17,26 \%)$, supermercados $(16,37 \%)$, otras ferias $(13,27 \%)$ y mercados $(7,96 \%)$, por otra parte, también compran principalmente por el precio $(24,6 \%)$ y diversos (26,9\%). El “crédito” se mencionó en el $14,9 \%$ de las respuestas.

Tabla 2. Razones y productos mencionados por los consumidores

\begin{tabular}{|c|c|c|}
\hline & Total & $\%$ \\
\hline \multicolumn{3}{|l|}{ Razones } \\
\hline A ces sibilidade & 7 & 1,6 \\
\hline A mistad & 2 & 0,4 \\
\hline A tendimiento & 1 & 0,2 \\
\hline Costumbre & 2 & 0,4 \\
\hline Distracción/Entretenimiento/Ocio & 93 & 20,7 \\
\hline Gustos/Preferencias & 43 & 9,6 \\
\hline Horánio de Funcionamiento & 5 & 1,1 \\
\hline Necesidad/Trabajo & 29 & 6,4 \\
\hline Precio & 145 & 32,2 \\
\hline Proximidad & 23 & 5,1 \\
\hline Calidad & 8 & 1,8 \\
\hline Otros & 5 & 1,1 \\
\hline \multirow[t]{2}{*}{ Variedade } & 87 & 19,3 \\
\hline & 450 & 100,0 \\
\hline
\end{tabular}

Tabla 2 continúa en la página siguiente > 
Polis, Revista Latinoamericana, Volumen 14, No 41, 2015

\begin{tabular}{lcc}
\hline Itens & 7 & \\
Animales & 2 & 4,05 \\
Antenas & 15 & 1,16 \\
A paratos de sonido & 3 & 8,67 \\
Antículos para el hogar & 1 & 1,73 \\
Automóbiles & 9 & 0,58 \\
Bicicleta & 3 & 5,20 \\
CD/DVD & 21 & 1,73 \\
Celular & 18 & 12,14 \\
De todo & 6 & 10,40 \\
Eletrodomésticos & 22 & 3,47 \\
Eletrónicos & 18 & 12,72 \\
Géneros Alimentícios & 10 & 10,40 \\
Herramientas & 1 & 5,78 \\
Libros & 2 & 0,58 \\
Muebles & 7 & 1,16 \\
Piezs de Automóbiles & 3 & 4,05 \\
Relojes & 3 & 1,73 \\
Televisión & 20 & 2,89 \\
Vestuario & 173 & 11,56 \\
\hline & & 100,00 \\
\hline
\end{tabular}

Fuente: Elaboración propia.

La Tabla 3 muestra los resultados del análisis factorial exploratorio de la escala de valores de compra. Se obtuvieron dos factores, hedónico y utilitario que en conjunto explican el $43,4 \%$ de la varianza total. La confiabilidad, medida por el Alpha de Cronbach, fue de 0,75 y 0,65 para cada factor, respectivamente. Los resultados del análisis factorial exploratorio son consistentes con los resultados obtenidos en otros estudios con grupos similares en la misma región (Peñaloza et al., 2012). 


\section{Tabla 3. Matriz de componentes rotacionada de los valores de compra}

\begin{tabular}{lcc}
\hline & \multicolumn{2}{c}{ Factores } \\
Variables & Hedónico Utilitario \\
\hline Fazer esta compra foi uma verdadeira distração para mim. & 0,70 \\
Eume diverti fazendo esta compra. & 0,70 \\
Comparando com outras coisas que eu poderia ter feito, o tempo gasto & 0,66 \\
comprando este produto foi muito mais agradável. & 0,62 \\
Estar na Feira foi um jeito muito agradável de passar o tempo. & 0,59 \\
A busca por este produto me deu um grande prazer. & 0,58 & \\
Esqueci os meus problemas enquanto estive na Feira. & 0,55 & 0,81 \\
Eu fiz esta compra porque quis e não por obrigação. & & 0,79 \\
Eu gastei apenas o tempo necessário para fazer esta compra. & & 0,58 \\
Eu fiz esta compra com rapidez. & & 0,47 \\
Foi fácil fazer esta compra porque eu sabia exatamente o que eu queria & 0,47 \\
comprar. & 27,83 \\
Fazer esta compra foi como cumprir uma obrigação. & 15,6 \\
Eu fui eficiente ao fazer esta compra. & 0,75 & 0,65 \\
\hline Varianza Explicada (\%) & & \\
Alpha de Cronbach & & \\
\hline Extraction Method: Principal Component Analysis. & \\
Rotation Method: Varimax with Kaiser Normalization. & & \\
a. Rotation converged in 3 iterations. & & \\
\hline
\end{tabular}

Fonte: Elaboración própria.

Utilizando las puntuaciones de los factores y haciendo la diferencia entre ellos, se observó que en el 52,8\% de la población entrevistada predomina el valor de compra utilitario. Sin embargo, la prueba t para igualdad de medias $(\mathrm{t}(114,13)=-0,63, ?=0,950))$ indica que no existen diferencias entre las clases sociales en el caso del consumo hedónico. En el caso del valor de compra utilitario $(\mathrm{t}(118,43)=-2,13$, ? $=0,036)$ ), las diferencias de medias son significativas, lo que indica que las clases sociales D y E, cuyos los vencimientos van hasta $4 \mathrm{sm}$, tienen un valor medio de compra significativamente mayor que el de las clases A, B y C, las cuales poseen vencimientos arriba de 4 sm; clasificación embazada en la división utilizada por la Associação Brasileira de Empresas de Pesquisa (2012).

\section{Consideraciones Finales}

El propósito de este trabajo fue el de contribuir al conocimiento del consumidor de mercados alternativos, mediante un estudio de caso en la Feria de los Pájaros, Brasil. Si bien es claro que la investigación presenta algunas limitaciones dadas por el muestreo no probabilístico, que hace que sus resultados no sean totalmente generalizables a todos los mercados no tradicionales, ni a todas las poblaciones de consumidores de baja renta. Este presenta algunas pistas que permiten aproximarse al conocimiento del perfil de las personas que acuden a los merca- 
dos alternativos de los cuales existen poquísimas informaciones disponibles.

En nuestro estudio, se aprecia una complementación entre una mirada cuantitativa y el rescate de los significados cualitativos, mostrándose consistencia en los resultados de ambas aproximaciones pero a la vez permite complementar aspectos que estaban velados y no se expresaban abiertamente en las respuestas a las preguntas directas.

Los resultados principales del abordaje cuantitativo muestran que efectivamente el público que asiste a la Feria de los Pájaros pertenece a los sectores de baja renta donde un 75,5\% de los encuestados pertenecen a las clases D y E, y 96,2\% no exceda de la clase C, 50\% de las familias ganan hasta 3 salarios mínimos y 40,2\% tienen sólo educación primaria o menos. Asimismo, se muestra que en su mayoría viven en lugares próximos o de fácil acceso en autobús y que los productos más buscados son celulares y artículos electrónicos. Otro dato interesante revela que el público que asiste la feria lo hace sobre todo por los bajos precios y la variedad, pero también en gran parte como una opción de diversión y recreación. No obstante ello, en términos globales sigue primando la compra utilitaria por sobre la hedónica.

La presencia de comportamientos de consumo hedonista y utilitarista, fueron evidenciados de manera más clara en el estudio cualitativo. En este, la categoría. Diversión/Entretenimiento es para los sujetos una representación fundamental de la Feria de los Pájaros. Así, la dimensión funcional, muestra la coexistencia de lo festivo y lo económico como rasgos de identidad en la caracterización de la feria. De hecho, este es un hallazgo importante para ser considerado cuando se montan espacios de consumo para estos sectores de baja renta, donde la posibilidad de diversión crea un valor agregado importante en el momento de decidir el lugar donde se harán las compras. Otro elemento subjetivo que emerge es el relacionado con los aspectos ilegales e informales de la Feria.

Reunidos en la dimensión estructural, de acuerdo con la categorización de Sherry (1990), el tema de la informalidad/desorganización, así como la inseguridad/miedo aparecen bastante citados (con una frecuencia superior al promedio), pero a pesar de ello, la gente no le atribuye tanta importancia (OME menor que el promedio), como a las categorías de dimensión funcional. El asunto de la ilegalidad, por ejemplo, se presenta como una categoría periférica y con connotaciones mucho más sutiles de lo esperado dado el conocimiento popular. Así, aparece más en el sentido de estafa o engaño, que en el sentido de algo ilícito o peligroso para la integridad física o económica del consumidor.

Por ello, son también periféricos en la representación de los consumidores aspectos como multitud, estrechez u otros adjetivos negativos atribuidos a la Feria, siendo ampliamente superados por los atributos positivos. Esto implica que la representación social de la Feria como un lugar 
inseguro o de delincuencia elevada no se derivaría necesariamente de la cultura local de quienes asisten a ella, sino que respondería a asignaciones exógenas incluyendo prejuicios que generan una estigmatización que viene del entorno externo a la Feria y que muchas veces es fomentada por las estrategias de posicionamiento de otros centros tradicionales como los shoppings. Por ello, surge la necesidad de investigación posterior sobre este tema contrastando con las estadísticas oficiales de actividades delictuales en el sector.

Un aspecto muy interesante radica en algunos de los rasgos atribuidos por los clientes de la Feria al tipo de contacto comercial que en ella se establece, rescatando un tipo de relación comercial más estrecha, respetuosa y amable, que constituiría una ventaja frente al trato despersonalizado del comercio de grandes superficies. En este sentido, las ferias parecen constituir un espacio que incorpora instancias de diálogo y socialización entre el cliente y quien lo atiende y también entre los propios compradores, lo que Salazar (2003) define como la recuperación parcial de la cultura social y el diálogo en un espacio abierto público en el que los participantes se sienten acogidos en sus necesidades no sólo de compra, sino fundamentalmente de relación recuperando su propia soberanía como ciudadanos consumidores en contraposición a los espacios de despersonalización propios de las grandes urbes (Páez, 2007). Llama así la atención, que en las ferias parece subsistir aquel tipo de relación que era propia del comercio de barrio, donde cliente y tendero compartían una relación tanto económica como de vinculación afectiva

Los resultados expuestos permiten aportar información que ayude a levantar la discusión en torno a la relevancia de los mercados alternativos o informales, los que a pesar de ser considerado "de segunda clase", constituyen una forma de comercialización importante y valorizada por las clases más populares, un segmento que puede constituir un importante nicho de mercado emergente.

Para el sector empresarial, el presente trabajo pretende contribuir aportando información para mejorar el conocimiento de este consumidor y de sus preferencias de consumo. En esta óptica, los espacios públicos como las ferias libres en lugares de menor desarrollo, podrían constituir una oportunidad no sólo a nivel de políticas públicas que garantizaran la permanencia de estos lugares como forma de alternativas de sobrevivencia para los comerciantes y como espacios de convivencia, entretenimiento y diversión para la población que las frecuenta sino también como una interesante reflexión para el sector privado. El comercio a pequeña escala, la atención personalizada y el cultivo del vínculo con el cliente son una enseñanza que se deriva de la observación de la realidad de las ferias y que puede ser un referente importante para la planeación de negocios con estos sectores emergentes. 


\section{Bibliografía}

Associação Brasileira de Empresas de Pesquisa. Critério de classificação econômica Brasil. Acceso en: 25 de Julio, 2014, disponible en: http:// www.abep.org/novo/Content.aspx?Content ID=301.

Babin, B. J., Darden, W. R. \& Griffin, M. (1994), "Work and/or Fun: Measuring Hedonic and Utilitarian Shopping Value”, The Journal of Consumer Research, Vol. 20, núm. 11, p. 644-656.

Belk, R., Wallendorf, M. \& Sherry, J. (1988), “A naturalistic Inquiry into buyer and seller behavior at a Swap Meet”, Journal of Consumer Research, Vol. 14, núm. 4, p. 449-470.

Calderon, F. (2012), "Las ferias medievales y su aporte al derecho comercial”, Revista Judicial, Costa Rica, núm. 106, Diciembre, p. 111-126.

Companion, M. (2008), “The underutilization of street markets as a source of food security indicators in Famine Early Warning Systems: a case study of Ethiopia”. Disasters, Vol. 32, núm. 3, p. 399-415.

Cuellar, M. C. \& Parra, C. (2001), "Las ferias medievales, origen de documentos de comercio”, In González, D. P.; Ramos, E. R.; Plaza, D. J.; Talavera, A. C. (Orgs.), Écrire, traduire et représenter la fête, Universitat de València,Valência.

Editora Verdes Mares (2011, Agosto 25), Diário do Nordeste: “Feira de ilegalidades todo fim de semana”, Fortaleza, CE.

Hair J. F., Black W. C., Babin B. J., Anderson R. E. \& Tatham R. L. (2005), Análise Multivariada de Dados, 5 a Edição, Bookman, Porto Alegre.

Hausman, A. (2000), “A multi-method investigation of consumer motivations in impulsive buying behavior”, Journal of Consumer Marketing, Vol. 17, núm. 5, p. 403-419.

Hemais, M. W., Casotti, L. M \& Rocha, E. P. J. (2010), “Hedonismo e Moralismo no Incentivo ao Consumo na Base da Pirâmide: Discussão para a Proposta de Uma Agenda Inicial de Pesquisa”, Anais do XXXIV Encontro Anual da ANPAD, 1, 803.

Hernandez, J. M. (2009), “Foi Bom para Você? Uma Comparação do Valor Hedônico de Compras Feitas em Diferentes Tipos de Varejistas”, Revista de Administração Mackenzie, Vol. 10, núm. 2, p. 11-30.

Holbrook, M. B. \& Hirschman, E. C. (1982), “The Experiential Aspects of Consumption: Consumer Fantasies, Feelings, and Fun”, The Journal of Consumer Research, Vol. 9, núm. 2, p. 132-140. 
Lauermann, J. (2012), "Performing Development in Street Markets: Hegemony, Governmentality, and the Qat Industry of Sana’a, Yemen”. Antipode, Vol. 44, núm. 4, p. 1329-1347.

Lopes, E. L., Teixeira, J. M., Hernandez, J. M. \& Moretti, S. L. (2010), "Valores de Compra Hedônico e Utilitário: Duas Aplicações no Varejo Especializado”, Anais do XXXIV Encontro Anual da ANPAD, 1, 803.

Molina, E. C. (2009), “Aportes conceptuales sobre las prácticas sociales en la feria callejera. El tema de la confianza social”. Revista Latinoamericana Polis, núm. 24, p. 2-12.

Olavarrieta, S., Manzur, E., Hidalgo, P. \& Farías, P. (2008), “Un análisis a los atributos relevantes de los mercados de las pulgas para los compradores: Evidencia desde América Latina”, Revista de Ciencias Sociales, Vol. 14, núm. 3, p. 468-478.

Pandolfo, M. L. (1987), “Feira de São Cristovão. A reconstrução do nordestino num mundo de paraíbas e nortistas”, Dissertação de Mestrado, Fundação Getúlio Vargas, Rio de Janeiro, RJ, Brasil.

Peñaloza, V., Gordiano, E. C. S \& Quezado, I. (2011), “Consumo Utilitario y Consumo Hedónico en el Beco da Poeira”, In XLVIAsamblea Anual de CLADEA (Consejo Latinoamericano de Escuela de Administración). San Juan, Porto Rico.

Prahalad, C. K. \& Hart, S. (2002), “The fortune at the bottom of the pyramid”, Strategy + Business, núm. 26, p. 54-67. Extraido el 2 Junio 2011, de http://www.cs.berkeley.edu/ brewer/ict4b/Fortune-BoP.pdf.

Rocha, A. \& Silva, J. C. (2009), “Consumo na Base da Pirâmide - Estudos Brasileiros”, Mauad X, Rio de Janeiro.

Salazar, G. (2003), "Ferias Libres: espacio residual de soberania ciudadana”, Editora SUR, Santiago.

Sherry, J. F. Jr. (1990), “Sociocultural Analysis of a Midwestern American Flea Market reviewed”. Journal of Consumer Research, Vol. 17, núm. 1, p. 13-30.

Vergara, S. (2008), Métodos de pesquisa em administração, $3^{\mathrm{a}}$ Edición, Atlas, São Paulo.

Zinkhan,G. M., Fontenelle, S. M. \& Balazs, A. L. (1999), “The structure of São Paulo street markets: envolving patterns of retail institutions”. The journal of consumer affairs, Vol. 33, núm. 1, p. 3-26. 